\title{
Association between DRD2/DRD4 Interaction and Conduct Disorder: A Potential Developmental Pathway to Alcohol Dependence
}

\author{
Mota, N. R., ${ }^{1,2}$ Bau,C. H. D., ${ }^{1}$ Banaschewski, T., ${ }^{3}$ Buitelaar, J. K., ${ }^{4}$ Ebstein, R. P., 5 Franke, \\ B., ${ }^{6,7}$ Gill, M., ${ }^{8}$ Kuntsi, J., ${ }^{2}$ Manor, I., ${ }^{9}$ Miranda, A., ${ }^{10}$ Mulas, F., ${ }^{11}$ Oades, R. D., ${ }^{12}$ Roeyers, H., ${ }^{13}$ \\ Rothenberger, A., ${ }^{14}$ Sergeant, J. A., ${ }^{15}$ Sonuga-Barke, E. J., ${ }^{13,16}$ Steinhausen, H-C., ${ }^{17,18,19}$ \\ Faraone, S. V., ${ }^{20,} 21$ and Philip Asherson, P. ${ }^{2}$
}

\section{American Journal of Medical Genetics Part B, 162B, 546-549.}

We present the pre-publication text. The definitive version (cited above) is available at http://onlinelibrary.wiley.com/DOI 10.1002/ajmg.b.32179/pdf

1 Department of Genetics, Universidade Federal do Rio Grande do Sul, Porto Alegre, Brazil

2 King's College London, Institute of Psychiatry, MRC Social Genetic and Developmental Psychiatry Centre, London, United Kingdom, UK

3 Department of Child and Adolescent Psychiatry and Psychotherapy, Central Institute of Mental Health, Medical Faculty Mannheim,

University of Heidelberg, Mannheim, Germany

4 Department of Cognitive Neuroscience, Donders Institute for Brain, Cognition and Behavior, Radboud University Medical Center, Nijmegen,

The Netherlands

5 Psychology Department, National University of Singapore, Singapore, Singapore

6 Department of Psychiatry, Donders Institute for Brain, Cognition and Behavior, Radboud University

Medical Center, Nijmegen, The Netherlands

7 Department of Human Genetics, Radboud University Medical Center, Nijmegen, The Netherlands

8 Department of Psychiatry, Trinity Centre for Health Sciences, St. James's Hospital, Dublin, Ireland

9 ADHD Unit, Geha Mental Health Centre, Petach-Tiqva, Israel

10 Departments of Developmental and Educational Psychology, University of Valencia, Valencia, Spain

11 Neuropediatrics Unit, La Fe' University Hospital, Valencia, Spain

12 University Clinics for Child and Adolescent Psychiatry, Essen, Germany

13 Department of Experimental Clinical and Health Psychology, Ghent University, Ghent, Belgium

14 Child and Adolescent Psychiatry, University of Göttingen, Göttingen, Germany

15 Department of Clinical Neuropsychology, Vrije Universiteit, Amsterdam, The Netherlands

16 Institute for Disorder on Impulse and Attention, School of Psychology, University of Southampton,

Southampton, United Kingdom, UK

17 Department of Child and Adolescent Psychiatry, University of Zurich, Zurich, Switzerland

18 Clinical Psychology and Epidemiology, Institute of Psychology, University of Basel, Basel, Switzerland

19 Research Unit for Child and Adolescent Psychiatry, Psychiatric Hospital, Aalborg University Hospital,

Aalborg, Denmark

20 Department of Neuroscience, SUNY Upstate Medical University, Syracuse, New York

21 Department of Psychiatry, SUNY Upstate Medical University, Syracuse, New York

Grant sponsor: National Institutes of Health; Grant numbers: R01MH62873, R01MH081803; Grant sponsor: UK Medical Research Council; Grant number: G03001896; Grant sponsor: CAPES Foundation, Ministry of Education of Brazil.

\section{Correspondence:}

Nina Roth Mota, M.Sc., Department of Genetics, Universidade Federal do Rio Grande do Sul, Porto Alegre, RS, Brazil. E-mail address: ninarothmota@gmail.com Prof. Philip Asherson, MRC Social Genetic and Developmental Psychiatry, Institute of Psychiatry, Kings College London, London SE 58AF, UK. E-mail address: philip.asherson@kcl.ac.uk 
In a recent article we reported the association of alcohol dependence with interacting genotypes of the dopamine D2 (DRD2) and D4 (DRD4) receptor genes [Mota et al., 2013]. The interaction between the two dopamine receptor genes is thought to reflect different DRD2-DRD4 heteromerization patterns, previously shown by experimental studies [Borroto-Escuela et al., 2011; Gonzalez et al., 2012]. The present study extends the investigation of the DRD2DRD4 interaction to behavioral phenotypes during childhood and adolescence known to contribute to the development of drug and alcohol dependence in adulthood.

Childhood attention deficit hyperactivity disorder (ADHD) predicts a higher prevalence of substance use disorder (SUD) in adulthood [Charach et al., 2011] and comorbid conduct disorder (CD) plays a mediating role in the association of ADHD with both SUD in general [Brook et al., 2010] and alcohol dependence specifically [Tuithof et al., 2012]. It is therefore possible that the reported DRD2-DRD4 association with adult alcohol dependence could be mediated by $\mathrm{CD}$, suggesting $\mathrm{a}$ developmental role for the DRD2-DRD4 interaction effect on early behavioral precursors of alcohol dependence.

To investigate this hypothesis we used a subset of the International Multicenter ADHD Genetics (IMAGE) project, for which the same DRD2 and DRD4 polymorphisms (rs2283265 and $48 \mathrm{bp}$-VNTR, respectively) that had been used in the previous report were available [Mota et al., 2013]. The sample comprised 559 unrelated children and adolescents with a research diagnosis of DSM-IV combined subtype ADHD, of which 142 had comorbid CD; with a mean age of 10.86 years $(S D=2.692$, range 7-17). Details of the sample, ascertainment, diagnostic procedures and genotyping have been reported previously [Brookes et al., 2006].
The DRD2 and DRD4 polymorphisms investigated in the present study are both thought to have functional effects and to interact with each other. In brief, the expression ratio of the short-(D2S) and long-(D2L) DRD2 isoforms (mainly presynaptic and postsynaptic, respectively) are affected by the DRD2 rs2283265 SNP, where the minor allele (T) favours the expression of the D2L form. This allele has been associated with cocaine abuse [Moyer et al., 2011] and reduced working memory and attention control performances [Zhang et al., 2007]. For the DRD4 48-bp variable number tandem repeat (VNTR) polymorphism in exon 3 , the most common variants are the 2-, 4- and 7repeat alleles $(2 R, 4 R$, and $7 R$, respectively), with $7 R$ being implicated in ADHD [Li et al., 2006].

Subjects were classified according to the presence of the DRD2 andDRD4 risk alleles ( $\mathrm{T}$ and $7 \mathrm{R}$, respectively) and, as in the previous study, subjects with rare DRD4 alleles (not 2R, 4R, or 7R) were excluded from all analyses; all $\mathrm{P}$-values are two-tailed. Chi-squared tests were first used to test the association of each SNP separately with CD. The presence of the T allele of the DRD2 rs2283265 SNP was associated with higher prevalence of $\mathrm{CD}(\mathrm{X} 2=6.628, \mathrm{P}=0.01)$, but there was no effect of the DRD4 7R allele (X2 = $0.073, P=0.79$ ). Logistic regression revealed a significant DRD2-DRD4 interaction (P 1/4 0.041) associated with CD (Table Ia). Estimation of the odds ratio (OR) in the presence of interaction was performed as previously described [Mota et al., 2013].

The findings indicated that the presence of the DRD2 T allele conferred an increased risk of $\mathrm{CD}\left(\mathrm{OR}_{\mathrm{CI}-95}=2.33\right.$ (1.41-3.86)) for subjects that did not have the DRD4 7R allele, whereas there was no risk associated with the DRD2 T allele for DRD4 7R carriers (OR CI_95 $=$ 0.96 (0.48-1.91)) (Table Ib). This DRD2DRD4 interaction pattern is similar to 
the previously reported association with alcohol dependence in adults with and without ADHD [Mota et al., 2013]. In the earlier study, the concomitant presence of both DRD2 $\mathrm{T}$ and DRD4 7R alleles was associated with a protective effect, compared to the presence of either one of them alone (i.e., in the absence of the other). Although in the present study no significant protective effect by the presence of both alleles was observed, the current results are in line with the previous study as they suggest that the significantly increased risk conferred by the DRD2 $\mathrm{T}$ allele is suppressed by the concomitant presence of the DRD4 7R risk allele.

It has been shown that DRD2 and DRD4 receptors can be colocalized in the prefrontal cortex and striatal regions, where they may be pre- and postsynaptically located [Svingos et al., 2000; Rivera et al., 2002; De Mei et al., 2009; De Almeida Mengod, 2010]. Experimental studies on DRD2-DRD4 heteromerization show that the D2L variant is able to heteromerize with the three main DRD4 variants, although less effectively with the DRD4 7R [BorrotoEscuela et al., 2011]. These authors suggest an enhancing allosteric

TABLE I. Interaction Effects Between DRD4 48bp VNTR and DRD2 rs2283265
Polymorphisms in Conduct Disorder

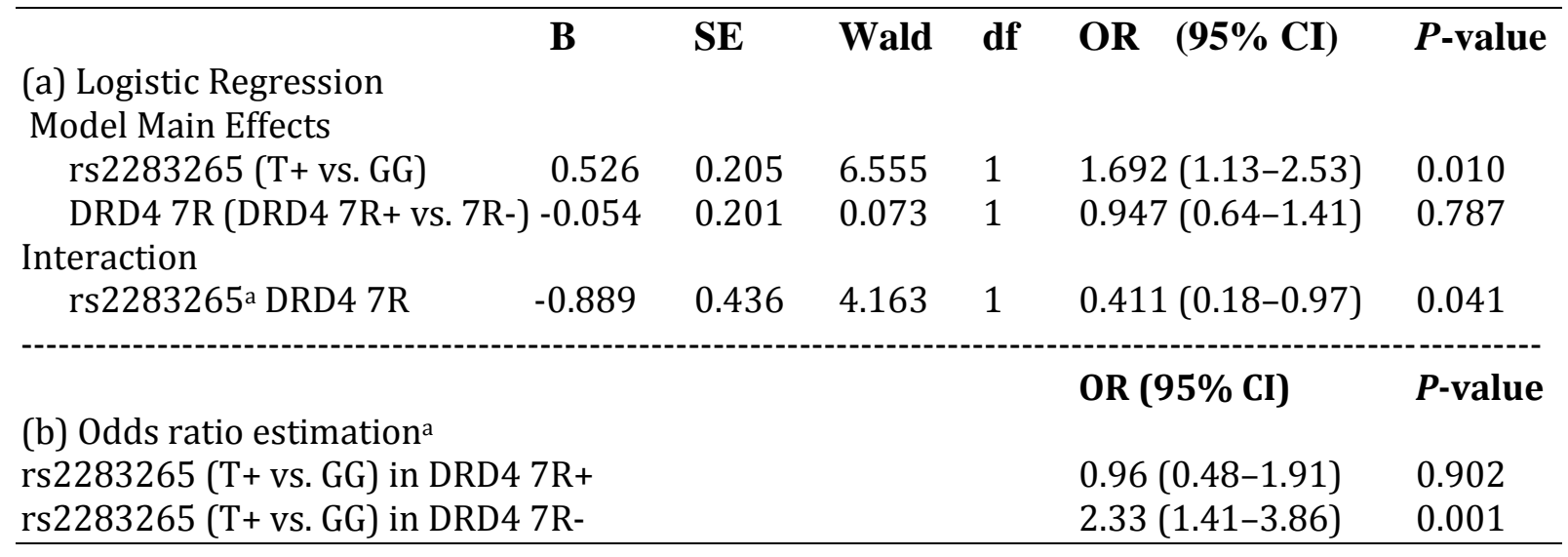

a Odds ratio estimation and corresponding confidence intervals $(95 \% \mathrm{CI})$ for the presence of DRD2 rs2283265 T allele (T+ vs. GG) in DRD4 7R carriers (DRD4 7+) and non-carriers (DRD4 7R-).

receptor-receptor interaction exists with the D2L-DRD4 2R and 4R heteromers, but not betweenD2L and DRD4 7R and that these different processes may play a role in the forebrain. Meanwhile, the D2S isoform is able to form heteromers only with DRD4 $2 \mathrm{R}$ and $4 \mathrm{R}$ receptor types, but not with the DRD4 7R variant [Gonzalez et al., 2012]. Based on in vivo and in vitro results, the authors strongly suggest that these D2S-DRD4 heteromers may have a key role in dopamine-mediated modulation of striatal glutamate release.

Although the present study does not test heteromerization patterns directly, it is possible that our results may be reflecting the expected different DRD2DRD4 heteromerization outcomes according to the concomitant presence (or absence) of the DRD4 7R, which is not able to heteromerize (or does it less efficiently) with D2S and D2L isoforms [Borroto-Escuela et al., 2011; Gonzalez 
et al., 2012]. Further interpretation of these results requires additional studies to clarify how the different DRD2-DRD4 heteromerization processes modulate dopaminergic and/or glutamatergic neurotransmission and whether any such functional changes explain the DRD2-DRD4 genetic associations reported in these studies.

A limitation of our study is the reliance on the candidate gene approach and the use of an interaction term, increasing the chances of a type I error. However within this context there is no substitute for replication and it is therefore of some interest that we see similar genetic effects on related externalizing phenotypes. Our previous study [Mota et al., 2013] implicated a significant DRD2-DRD4 interaction effect in alcohol dependence in two independent samples. In the present study we were able to demonstrate that such interaction is also associated with CD in a sample of children/adolescents with ADHD. Despite the above stated limitations and differences between the two studies, the findings of the DRD2-

DRD4 interaction association with childhood/adolescent CD, a frequent precursor of later SUD, suggest a potential mechanism for one possible causal pathway that links childhood CD to the later development of alcohol dependence.

In summary, this is the third independent sample where disorders

\section{References:}

Borroto-Escuela D0, Van Craenenbroeck K, Romero-Fernandez W, Guidolin D, Woods AS, Rivera A, Haegeman G, Agnati LF, Tarakanov AO, Fuxe K. 2011. Dopamine D2 and D4 receptor heteromerization and its allosteric receptor-receptor interactions. Biochem Biophys Res Commun 404: 928-934.

Brook DW, Brook JS, Zhang C, Koppel J. 2010. Association between attention- related to externalizing behavior are found to be associated with a DRD2DRD4 interaction term. While these results look highly promising, further replication is still required to clarify the role of the DRD2-DRD4 interaction on the course and outcome of childhood behavioral disorders on adult alcohol dependence, and to establish the processes involved throughout development.

Acknowledgments: The IMAGE project was supported in part by National Institutes of Health (NIH) grants R01MH62873 and R01MH081803 to S.V.F. and, in London, by a UK Medical Research Council grant G03001896 to J.K. This work was also supported by a fellowship from CAPES Foundation, Ministry of Education of Brazil.

Conflicts of interest: Consultancy fees were received by $\mathrm{PA}, \mathrm{TB}$, and $\mathrm{AR}$ from Shire, Lilly, Novartis, and Medice; and TB from Bristol Myers-Squibb, Develco Pharma and Vifor Pharma; and PA and TB from Janssen. Speaker/travel fees were paid to PA and TB from Shire, Lilly, Novartis, Janssen, and Medice; and AR from shire. PA held educational or research grants from Janssen, Shire, Vifor and QBTech; and AR from Shire. The present work is unrelated to the above grants and relationships. No other conflicts of interest are noted.

deficit/hyperactivity disorder in adolescence and substance use disorders in adulthood. Arch Pediatr Adolesc Med 164: 930-934.

Brookes $\mathrm{K}, \mathrm{Xu} \mathrm{X}$, Chen W, Zhou K, Neale B, Lowe N, Anney R, Aneey R, Franke B, Gill M, Ebstein R, Buitelaar J, Sham P, Campbell D, Knight J, Andreou P, Altink M, Arnold R, Boer F, Buschgens C, Butler L, Christiansen H, Feldman L, Fleischman K, Fliers E, Howe-Forbes R, 
Goldfarb A, Heise A, Gabriels I, KornLubetzki I, Johansson L, Marco R, Medad $S$, Minderaa $R$, Mulas $F$, Müller $U$, Mulligan A, Rabin K, Rommelse N, Sethna V, Sorohan J, Uebel H, Psychogiou L, Weeks A, Barrett R, Craig I, Banaschewski T, Sonuga-Barke E, Eisenberg J, Kuntsi J, Manor I, McGuffin P, Miranda A, Oades RD, Plomin R, Roeyers H, Rothenberger A, Sergeant J, Steinhausen H-C, Taylor E, Thompson M, Faraone SV, Asherson P. 2006. The analysis of 51 genes in DSM-IV combined type attention deficit hyperactivity disorder: Association signals in DRD4, DAT1 and 16 other genes. Mol Psychiatry 11: 934-953.

Charach A, Yeung E, Climans T, Lillie E. 2011. Childhood attention-deficit/ hyperactivity disorder and future substance use disorders: Comparative meta-analyses. J Am Acad Child Adolesc Psychiatry 50: 9-21.

De Almeida J, Mengod G. 2010. D2 and D4 dopamine receptor mRNA distribution in pyramidal neurons and GABAergic subpopulations in monkey prefrontal cortex: Implications for schizophrenia treatment. Neuroscience 170: 1133-1139.

De Mei C, Ramos M, Iitaka C, Borrelli E. 2009. Getting specialized: Presynaptic and postsynaptic dopamine D2 receptors. Curr Opin Pharmacol 9: 5358.

Gonzalez S, Rangel-Barajas C, Peper M, Lorenzo R, Moreno E, Ciruela F, Borycz J, Ortiz J, Lluis C, Franco R, McCormick PJ, Volkow ND, Rubinstein M, Floran B, Ferre S. 2012. Dopamine D4 receptor, but not the ADHD-associated D4.7 variant, forms functional heteromers with the dopamine D2S receptor in the brain. Mol Psychiatry 17: 650-662.

Li D, Sham PC, Owen MJ, He L. 2006. Meta-analysis shows significant association between dopamine system genes and attention deficit hyperactivity disorder (ADHD). Hum Mol Genet 15: 2276-2284.

Mota NR, Rovaris DL, Bertuzzi GP, Contini V, Vitola ES, Grevet EH, Roman T, Callegari-Jacques SM, Hutz MH, Bau CHD. 2013. DRD2/DRD4 heteromerization may influence genetic susceptibility to alcohol dependence. Mol Psychiatry 18: 401-402.

Moyer RA, Wang D, Papp AC, Smith RM, Duque L, Mash DC, Sadee W. 2011. Intronic polymorphisms affecting alternative splicing of human dopamine D2 receptor are associated with cocaine abuse. Neuropsychopharmacology 36: 753-762.

Rivera A, Cuellar B, Giron FJ, Grandy DK, de la Calle A, Moratalla R. 2002. Dopamine D4 receptors are heterogeneously distributed in the striosomes/matrix compartments of the striatum. J Neurochem 80: 219-229.

Svingos AL, Periasamy S, Pickel VM. 2000. Presynaptic dopamine D(4) receptor localization in the rat nucleus accumbens shell. Synapse 36: 222-232.

Tuithof $M$, ten Have M, van den Brink W, Vollebergh W, de Graaf R. 2012. The role of conduct disorder in the association between ADHD and alcohol use (disorder). Results from the Netherlands Mental Health Survey and Incidence Study 2. Drug Alcohol Depend 123: 115-121.

Zhang Y, Bertolino A, Fazio L, Blasi G, Rampino A, Romano R, Lee M-LT, Xiao T, Papp A, Wang D, Sadee W. 2007. Polymorphisms in human dopamine D2 receptor gene affect gene expression, splicing, and neuronal activity during working memory. Proc Natl Acad Sci USA104: 20552-20557. 